\title{
A product space with the fixed point property
}

\author{
Helga Fetter Nathansky ${ }^{1 *}$ and Enrique Llorens-Fuster ${ }^{2}$
}

\footnotetext{
* Correspondence: fetter@cimat.mx ${ }^{1}$ Centro de Investigación en Matemáticas (CIMAT), Jalisco s/n, 36240 Guanajuato (Gto.), México Full list of author information is available at the end of the article
}

\begin{abstract}
We show that the $I_{1}$ sum of the van Dulst space with itself possesses the fixed point property, although it fails most of the known conditions that imply (FPP).
\end{abstract}

Mathematics Subject Classification: Primary, 46B20; 47H09.

Keywords: fixed point, asymptotic normal structure, E-convexity, (PS) condition, property WORTH, orthogonal convexity

\section{Introduction}

Let $C$ be a subset of a Banach space $(X,\|\cdot\|)$. A mapping $T: C \rightarrow X$ is called nonexpansive whenever $\|T x-T y\| \leq\|x-y\|$ for all $x, y \in C$. The space $(X,\|\cdot\|)$ has the fixed point property (FPP) if every nonexpansive self-mapping of each nonempty bounded closed convex subset $C$ of $X$ has a fixed point. If the same property holds for every weakly compact convex subset of $X$ we say that $(X,\|\cdot\|)$ has the weak fixed point property (WFPP for short).

It has been known from the outset of the study of this property (around the early sixties of last century) that it depends strongly on "nice" geometrical properties of the space. A seminal work for this theory, due to Kirk [1] established that those Banach spaces with normal structure (NS), have the (WFPP). In particular uniformly convex Banach spaces have normal structure. A long time open major question in metric fixed point theory is: Does every reflexive Banach space have (FPP)? (See [2] for more about this problem). A special case of this question is: Does every superreflexive Banach space have (FPP)? Although superreflexive spaces have the fixed point property for isometries [3] the question for general nonexpansive mappings remains still unsolved.

Since 1965 considerable effort has been made in order to find sufficient conditions for (WFPP). Maybe the most relevant of those geometrical conditions are the following (see definitions below). We indicate along with the name of each property the year it was proved that it implies (WFPP).

(1) Asymptotic normal structure (ANS) (1981).

(2) Orthogonal convexity (OC) (1993).

(3) Prus-Szczepanik condition (PS) (2005).

(4) E-convexity (2008).

(5) Property WORTH (2010). 
Each one of these properties is implied by other more or less known geometrical conditions. For instance, uniformly nonsquare Banach spaces satisfy both, (PS) and E-convexity, and uniform convexity implies (1), (2), (3) and (4).

Another seemingly open question in this field is whether (WFPP) is preserved by direct sums. Many partial affirmative answers have been obtained during the last three decades.

The aim of these notes is to give a product Banach space with the following characteristics:

(1) It enjoys (FPP).

(2) It fails all of the above conditions (1)-(5).

(3) It is the $l_{1}$ product of two Banach spaces with (FPP) but it falls out of the scope of all known results guaranteeing the (FPP) for such products.

\section{Sufficient conditions for (WFPP)}

From here on, we will use the standard notation in Banach space theory, in particular $B[x, r]$ denotes the closed ball with center $x \in X$ and radius $r>0 . B_{X}$ and $S_{X}$ denote the unit ball and the unit sphere in $X$. The weak convergence of the sequence $\left(x_{n}\right)$ to $x$ will be denoted by $x_{n} \underset{w}{\rightarrow}$.

\subsection{Asymptotic normal structure (ANS)}

This property was introduced by Baillon and Schöneberg [4].

Definition 1. A Banach space $(X,\|\cdot\|)$ has (ANS) if for every nonempty bounded, closed and convex subset $K$ of $X$ consisting of more than one point, and every sequence $\left(x_{n}\right) \subset K$ with $\lim _{n}\left(x_{n}-x_{n+1}\right)=0$, there exists $x \in K$ such that $\lim \inf _{n}\left\|x_{n}-x\right\|<$ $\operatorname{diamK}$.

(ANS) is weaker than normal structure (NS). Among many others, the following geometrical conditions in turn imply (NS):

(a) Uniform convexity (UC), (Belluce, Kirk, 1967).

(b) $\varepsilon_{0}(X)<1$. (Goebel, 1970). Here $\varepsilon_{0}(X)$ denotes the characteristic of convexity of $X$.

(c) Uniform smoothness (Turett, 1982).

(d) Uniform convexity in every direction (Garkavi, 1962).

(e) Opial condition (Gossez and Lami Dozo, 1972).

(f) Near uniform convexity (van Dulst, 1981).

Theorem 1. [4] Every reflexive Banach space with asymptotic normal structure has the $(F P P)$.

\subsection{Orthogonal convexity (OC)}

This property is independent of (ANS) and weaker than uniform convexity. It was introduced in 1988 (see [5,6]). James space $J, c_{0}, c$ and Banach spaces with the Schur property like $l_{1}$, are (OC). To define (OC) we need some further notation. For $x, y \in X$,

$M_{\beta}(x, y)=B\left[x, \frac{1+\beta}{2}\|x-y\|\right] \cap B\left[y, \frac{1+\beta}{2}\|x-y\|\right]$. 
If $A$ is a bounded subset of $X,|A|=\sup \{\|x\|: x \in A\}$.

If $\left(x_{n}\right)$ is a bounded sequence in $X, D\left[\left(x_{n}\right)\right]=\lim \sup _{m}\left(\lim \sup _{n}\left\|x_{m}-x_{n}\right\|\right)$.

Definition 2. A Banach space $(X,\|\|$.$) is orthogonally convex (OC) if for every weakly$ null sequence $\left(x_{n}\right)$ with $D\left[\left(x_{n}\right)\right]>0$, there exists $\beta>0$ such that

$$
\underset{n}{\limsup }\left(\limsup _{m}\left|M_{\beta}\left(x_{n}, x_{m}\right)\right|\right)<D\left[\left(x_{n}\right)\right] \text {. }
$$

Theorem 2. Every Banach space satisfying (OC) has (WFPP).

\subsection{Prus-Szczepanik condition (PS)}

This condition was introduced by Prus and Szczepanik [7]. Given a Banach space $X, x$ $\in X, \varepsilon>0$ put

$$
d(1, x)=\inf _{\left(y_{m}\right) \in \mathcal{N}_{X}} \lim \sup _{m}\left\|x+y_{m}\right\|-\|x\|,
$$

and

$$
b_{1}(1, x)=\sup _{\left(y_{m}\right) \in \mathcal{M}_{x}} \liminf \inf _{m}\left\|x+y_{m}\right\|-\|x\|,
$$

where

$$
\mathcal{N}_{X}=\left\{\left(x_{n}\right): x_{n} \in S_{X}, n=1,2, \ldots, x_{n} \underset{w}{\rightarrow} 0\right\}
$$

and

$\mathcal{M}_{X}=\left\{\left(x_{n}\right): x_{n} \in B_{X}, n=1,2, \ldots, D\left[\left(x_{n}\right)\right] \leq 1, x_{n} \underset{w}{\rightarrow} 0\right\}$.

Definition 3. Let $X$ be a non-Schur space. If there exists $\varepsilon \in(0,1)$ such that for every $x \in S_{X}$ it is the case that $b_{1}(1, x)<1-\varepsilon$ or $d(1, x)>\varepsilon$ we say that $(X,\|\|$.$) satisfies$ the (PS) condition.

Theorem 3. Every non-Schur reflexive Banach space satisfying (PS) condition has (FPP).

Properties stronger than (PS) condition are the following:

(a) Uniform convexity.

(b) Uniform noncreasyness, introduced by Prus (see [8]) and its generalizations (see [9-11]).

(c) Property $M(X)>1$ (see [12]). In particular this condition covers the uniformly nonsquare Banach spaces (see $[13,14])$. Other reflexive Banach spaces with $M(X)$ $>1$ are those satisfying $R(X)<2$ (see [15]).

\subsection{Conditions depending on the dual space}

Very recently the authors in [16] deduced that spaces with $O$-convex dual have the (FPP) from the result proved in [13] that $\tilde{\varepsilon}_{0}^{n}(X)<2$ implies that $X^{*}$ has the (FPP). The fact that E-convex spaces have the (FPP) was proved in a different manner in [17].

The geometrical properties of $\mathrm{O}$ and E-convexity were coined by Naidu and Sastry [18] who proved several consequences of these properties. For instance they showed that $\mathrm{O}$-convex spaces are superreflexive and that they include uniformly nonsquare Banach spaces. 
Definition 4. Let $0<\varepsilon<2, n \geq 2$. $(X,\|\cdot\|)$ is $O(n, \varepsilon)$ convex, if for every $x_{1}, x_{2}, \ldots$, $x_{n}$ in $B_{X}\left(S_{X}\right)$ there exist $i \neq j \in\{1, \ldots, n\}$ such that

$$
\min \left(\left\|x_{i}+x_{j}\right\|,\left\|x_{i}-x_{j}\right\|\right) \leq 2-\varepsilon .
$$

$X$ is $O(n)$ convex, if it is $O(n, \varepsilon)$ convex for some $\varepsilon>0$ and O-convex if it is $O(n)$ convex for some $n \in N$. A Banach space $X$ is E-convex if and only if its dual space $X^{*}$ is O-convex.

Theorem 4. $[17,16]$. If $X^{*}$ is O-convex, that is if $X$ is E-convex, then the Banach space $X$ has the fixed point property for nonexpansive mappings.

\subsection{Property WORTH}

A Banach space has the WORTH property (Rosenthal, 1983; Sims 1988) if $\lim _{n}|| \mid x_{n}$ $x\|-\| x_{n}+x \| \mid=0$ for all $x \in X$ and all weakly null sequences $\left(x_{n}\right)$ in $X$.

In [19] a coefficient was defined which in some sense quantifies how far a Banach space is from possessing this property. This coefficient is given by $\mu(X)=\inf \left\{r>0: \lim \sup _{n}\left\|x_{n}+x\right\| \leq r \lim \sup _{n}\left\|x_{n}-x\right\|,\left(x_{n}\right)\right.$ in $\left.X, x_{n} \underset{w}{\rightarrow} 0, x \in X\right\}$.

Clearly $\mu(X)=1$ if and only if $X$ has WORTH.

Sims raised the question whether reflexive spaces with WORTH property have (FPP). Recently Fetter and Gamboa [20] solved this problem. That is,

Theorem 5. (Fetter Gamboa 2010). If $X$ is reflexive and $\mu(X)=1$, then $X$ enjoys $(F P P)$.

\section{The van Dulst space}

Let $V$ be the space $l_{2}$ equipped with the following equivalent norm:

$$
\||x|\|=\max \left\{\frac{1}{3}\|x\|_{2}, \sup _{n \geq 1}|x(1)+x(n)+x(n+1)|: n \geq 2\right\} .
$$

This space was introduced in 1982 by van Dulst. It is (OC) [6] but it fails to have asymptotic normal structure [21]. Moreover, for every $x \in l_{2}$,

$$
\frac{1}{3}\|x\|_{2} \leq|\|x\|| \leq \sqrt{3}\|x\|_{2} \text {. }
$$

We enumerate several geometrical properties of this space in the following result.

Proposition 1. For the van Dulst space V, one has

(1) $V$ fails (ANS).

(2) $V$ is $(O C)$.

(3) $V$ fails (PS) condition.

(4) $V$ fails to have O-convex dual.

(5) $\mu(V) \geq 2$ and hence $V$ fails WORTH property.

Proof. See [21] for (1) and [6] to see (2).

To see (3), for every positive integer $n$ put

$$
x_{n}=\left(0 \ldots, 0, \frac{1}{2}, \frac{1}{2}, 0, \ldots\right) .
$$


Note that $\left|\left\|x_{n}\right\|\right|=1$ and that, for $m>n$,

$$
\left|\left\|x_{m}-x_{n}\right\|\right|=\left|\left\|\left(0, \ldots, 0,-\frac{1}{2},-\frac{1}{2}, 0, \ldots, 0, \frac{1}{2}, \frac{1}{2}, 0, \ldots\right)\right\|\right|=1 \text {. }
$$

Then $\left(x_{n}\right) \in \mathcal{M}_{V}=\left\{\left(y_{n}\right): y_{n} \in B_{V} n=1, \ldots, D\left(y_{n}\right) \leq 1, y_{n} \underset{w}{\rightarrow} 0\right\}$. Moreover, $e_{1} \in S_{V}$ and for every $n>1$,

$$
\left|\left\|e_{1}+x_{n}\right\|\right|=||\left(1,0, \ldots 0, \frac{1}{2}, \frac{1}{2}, \ldots\right) \| \mid=2
$$

Therefore, for every $\varepsilon \in(0,1)$,

$$
\begin{aligned}
b_{1}\left(1, e_{1}\right) & =\sup _{\left(y_{n}\right) \in \mathcal{M}_{V}} \liminf \inf _{n}\left|\left\|e_{1}+y_{n}\right\|\right|-\left|\left\|e_{1}\right\|\right| \\
& \geq \liminf _{n}\left|\left\|e_{1}+x_{n}\right\|\right|-\left|\left\|e_{1}\right\|\right| \\
& =2-1>1-\varepsilon .
\end{aligned}
$$

In the same way, if we consider the sequence $\left(-x_{n}\right)$, it is clear that $\left(-x_{n}\right) \in \mathcal{N}_{V}=\left\{\left(x_{n}\right): x_{n} \in S_{V} n=1,2, \ldots, x_{n} \underset{w}{\rightarrow} 0\right\}$. For $n>1$,

$$
\left|\left\|e_{1}-x_{n}\right\|\right|=\left|\left\|\left(1,0, \ldots 0,-\frac{1}{2},-\frac{1}{2}, \ldots\right)\right\|\right|=\max \left\{\frac{1}{3} \sqrt{\frac{3}{2}}, 1\right\}=1,
$$

and

$$
\begin{aligned}
d\left(1, e_{1}\right) & =\inf _{\left(y_{n}\right) \in \mathcal{N}_{V}} \lim \sup _{n}\left|\left\|e_{1}+y_{n}\right\|\right|-\left|\left\|e_{1}\right\|\right| \\
& \leq \lim \sup _{n}\left|\left\|e_{1}-x_{n}\right\|\right|-\left|\left\|e_{1}\right\|\right| \\
& =1-1=0<\varepsilon .
\end{aligned}
$$

Thus for every $\varepsilon \in(0,1) e_{1} \in S_{V}, b_{1}\left(1, e_{1}\right)>1-\varepsilon$ and $d\left(1, e_{1}\right)<\varepsilon$ which implies that $V$ fails condition (PS).

(4) To see that $V^{*}$ is not O-convex, consider for any positive integer $n$ the functional $f_{n} \in V^{*}$ defined on $V$ by $f_{n}(x)=x(1)+x(2 n)+x(2 n+1)$.

For every $x \in V$

$$
\left|f_{n}(x)\right| \leq|x(1)+x(2 n)+x(2 n+1)| \leq|\|x\|| .
$$

Moreover, $\left|\left\|e_{2 n}\right\|\right|=1$ and $f_{n}\left(e_{2 n}\right)=1$. Thus $\left\|f_{n}\right\|_{V^{*}}=1$.

Let $m>n$ and take $v_{m, n}=\frac{1}{2}\left(e_{2 n+1}+e_{2 n}-e_{2 m+1}-e_{2 m}\right)$, $w_{m, n}=\frac{1}{2}\left(e_{2 n+1}+e_{2 n}+e_{2 m+1}+e_{2 m}\right)$.

Clearly $\left|\left\|v_{m, n}\right\|\right|=\left|\left\|w_{m, n}\right\|\right|=1$. Since $\left(f_{n}-f_{m}\right)\left(v_{m, n}\right)=2,\left\|f_{n}-f_{m}\right\|_{V^{*}}=2$.

Therefore, $\mathcal{A}=\left\{f_{n}: n=1,2, \ldots\right\}$ is a countable 2 -separated subset of the unit sphere $S_{V^{*}}$. Moreover, $\left(f_{n}+f_{m}\right)\left(w_{m, n}\right)=2$ which implies that $\left\|f_{n}+f_{m}\right\|_{V^{*}}=2$. Thus $\mathcal{A}$ is a countable 2-symmetrically separated subset of $S_{V^{*}}$ and hence $V^{*}$ is not O-convex. 
Finally, to see (5) take $x_{n}$ as in (3.1). Then $\left(x_{n}\right)$ is weakly null and by (3.2) and (3.3), for $n>1,\left|\left\|e_{1}+x_{n}\right\|\right|=2$ and $\left|\left\|e_{1}-x_{n}\right\|\right|=1$. Since $e_{1} \in S_{V}$, it follows that $\mu(V) \geq 2$.

\section{The space $V \oplus_{1} V$}

In this section we will be concerned with the space $W=V \oplus_{1} V$, that is the product space $l_{2} \times l_{2}$ endowed with the norm

$$
\|(u, v)\|=|\|u\||+|\|v\|| .
$$

Now consider the space $Z$ to be $l_{2}$ with the following norm:

$$
\begin{aligned}
\|x\| & =\max \left\{\frac{1}{3}\left(\sum_{n=1}^{\infty} x(2 n)^{2}\right)^{\frac{1}{2}}, \sup _{n \geq 1}|x(2)+x(2 n+2)+x(2 n+4)|\right\}+ \\
& \max \left\{\frac{1}{3}\left(\sum_{n=1}^{\infty} x(2 n-1)^{2}\right)^{\frac{1}{2}}, \sup _{n \geq 1}|x(1)+x(2 n+1)+x(2 n+3)|\right\} .
\end{aligned}
$$

Let $\left(e_{n}\right)$ be the canonical basis of $l_{2}$ and $P x=\sum_{n=1}^{\infty} x(2 n) e_{2 n}$ and $\mathrm{Q} x=\sum_{n=1}^{\infty} x(2 n-1) e_{2 n-1}$. Then we may write

$$
\begin{aligned}
\|x\|= & \max \left\{\frac{1}{3}\|P x\|_{2}, \sup _{n \geq 1}|x(2)+x(2 n+2)+x(2 n+4)|\right\}+ \\
& \max \left\{\frac{1}{3}\|Q x\|_{2}, \sup _{n \geq 1}|x(1)+x(2 n+1)+x(2 n+3)|\right\} .
\end{aligned}
$$

This implies that

$$
\|x\|=\|P x\|+\|Q x\| .
$$

Since

$$
\frac{1}{3}\left(\|P x\|_{2}^{2}+\|Q x\|_{2}^{2}\right)^{\frac{1}{2}} \leq \frac{1}{3}\left(\|P x\|_{2}+\|Q x\|_{2}\right) \leq\|x\| \quad \text { and } \quad \text { since }
$$

$\sum_{i=1}^{n} a_{i} \leq \sqrt{n}\left(\sum_{i=1}^{n} a_{i}^{2}\right)^{\frac{1}{2}}$ we have

$$
\frac{1}{3}\|x\|_{2} \leq\|x\| \leq \sqrt{6}\|x\|_{2} .
$$

Clearly the function $F: Z \rightarrow W$ given by

$$
F(x)=\left(\sum_{n=1}^{\infty} x(2 n) e_{n}, \sum_{n=1}^{\infty} x(2 n-1) e_{n}\right)
$$

is an isometry between the two spaces. We will work mostly with the space $Z$.

Proposition 2. Let $W=V \oplus_{1} V$. Then

(1) W fails (ANS).

(2) W fails to be (OC).

(3) W fails (PS) condition.

(4) W fails to have O-convex dual.

(5) $\mu(W) \geq 2$ and hence $W$ fails WORTH property. 
Proof. Since the van Dulst space $V$ is isometric to a closed subspace of $W$, and asymptotic normal structure (ANS), Prus-Szczepanik condition (PS) and WORTH are inherited by closed subspaces, $W$ fails (ANS), (PS) and WORTH.

For every $n \in \mathbb{N}$ let $F_{n} \in Z^{*}$ be given by $F_{n}(x)=x(1)+x(2 n+1)+x(2 n+3)$. Then, since $F_{n}\left(e_{1}\right)=1$ and clearly $\left\|F_{n}\right\|_{Z^{*}} \leq 1$, we have that $\left\|F_{n}\right\|_{Z^{*}}=1$. If $w_{m, n}=\frac{1}{2}\left(e_{2 n+1}+e_{2 n+3}+e_{2 m+1}+e_{2 m+3}\right), w_{m, n}=\frac{1}{2}\left(e_{2 n+1}+e_{2 n+3}+e_{2 m+1}+e_{2 m+3}\right)$ for $n \neq$ $m$, then $\left\|v_{m, n}\right\|=\left\|w_{m, n}\right\|=1$ and $\left(F_{n}-F_{m}\right)\left(v_{m, n}\right)=\left(F_{m}+F_{n}\right)\left(w_{m, n}\right)=2$; thus $\left\|F_{n}-F_{m}\right\|_{Z^{*}}=\left\|F_{n}+F_{m}\right\|_{Z^{*}}=2$.

Hence $\left\{F_{n}: n=1,2, \ldots\right\}$ is a symmetrically separated subset of $S_{Z^{*}}$, the space $Z^{*}$ cannot be O-convex and statement (4) is proven.

On the other hand consider the weakly null sequence $\left(e_{k}\right)$ in $Z$. It is obvious that $\|$ $e_{k} \|=1$, that $\left\|e_{n}-e_{m}\right\| \leq 2$ for $n \neq m$ and that $\left\|e_{n}-e_{m}\right\|=2$ whenever $m$ and $n$ have different parity. Thus $D\left(e_{n}\right)=\lim \sup _{m}\left(\lim \sup _{n}\left\|e_{n}-e_{m}\right\|\right)=2$. If $\beta>0$, and $m$ and $n$ have different parity, since

$$
\left\|\left(e_{m}+e_{n}\right)-e_{n}\right\|=1=\frac{1}{2}\left\|e_{n}-e_{m}\right\|=\left\|\left(e_{m}+e_{n}\right)-e_{m}\right\|,
$$

we have that $e_{m}+e_{n} \in M_{\beta}\left(e_{m}, e_{m}\right)$. Since $\left\|e_{n}+e_{m}\right\|=2$, then

$$
\left|M_{\beta}\left(e_{n}, e_{m}\right)\right| \geq\left\|e_{m}+e_{n}\right\|=2
$$

and therefore for every $\beta>0$,

$$
\lim \sup _{m}\left[\lim \sup _{n}\left|M_{\beta}\left(e_{n}, e_{m}\right)\right|\right] \geq 2=D\left[\left(e_{n}\right)\right] .
$$

which implies that $W$ fails to be $(O C)$, that is, statement (2) holds. This completes the proof.

Remark 1. It seems to be an open problem whether $X_{1} \oplus_{1} X_{2}$ has the (WFPP) provided that $X_{1}, X_{2}$ are two Banach spaces with the (WFPP). There are several affirmative partial answers in the literature. For instance in [22] it was shown that $X_{1} \oplus_{1} X_{2}$ enjoys (WFPP) whenever $X_{1}$ is uniformly convex in every direction and $X_{2}$ is a Banach space such that $\mathbb{R} \times X_{2}$ with the $l_{1}$-norm has the (WFPP). In the same paper it was pointed out that this last condition holds whenever $X_{2}$ has either uniformly normal structure or many other well known sufficient conditions for (FPP).

In 2002, A. Wiśnicki proved that $X_{1} \oplus_{1} X_{2}$ has the (WFPP) provided that $X_{1}$ and $X_{2}$ are uniformly noncreasy Banach spaces. The notion of uniformly noncreasy spaces was introduced by S. Prus to describe a large class of superreflexive Banach spaces with the (WFPP).

In [23] it was shown that $X_{1} \oplus_{1} X_{2}$ has the (WFPP) provided that $X_{1}$ is a Banach space with both, the weak Banach-Saks property and the (WFPP) while $X_{2}$ has asymptotic (P). Asymptotic $(P)$ is a geometric property which implies normal structure, which in turn implies (ANS) and (WFPP), introduced by B. Sims and M.A. Smyth in 1995. Very recently in [24], A. Wiśnicki proved that the assumption of the Banach-Saks property could be dropped in the above result.

The van Dulst space $V$ which has the weak Banach-Saks property and (WFPP), does not have normal structure. Hence it fails uniform normal structure and asymptotic (P). 
(In fact it fails (ANS) which is even weaker than (NS)). Moreover, $V$ is not uniformly noncreasy, because it fails (PS) condition which is more general than uniformly noncreasyness.

Thus we can see that for the space $W=V \oplus_{1} V$, (WFPP) is preserved beyond the scope of the above theorems regarding the (WFPP) of $l_{1}$ direct sums.

So we will turn our attention to show that $Z$ and thus $W$ has the (FPP). The proof is rather technical and we first need the following lemma which was first proved by Maurey [25].

We recall that if $T: K \rightarrow X$ where $K \subset X$, a sequence $\left(x_{n}\right)$ in $K$ is called almost fixed point (afp) sequence (afps) for $T$ provided that $\left\|x_{n}-T x_{n}\right\| \rightarrow 0$.

Lemma 1. Let $X$ be a Banach space and $T: K \rightarrow K$, where $K \subset X$ is a weakly compact convex set, be a nonexpansive mapping. If $\left(x_{n}^{1}\right)$ and $\left(x_{n}^{2}\right)$ are afp sequences for $T$, then there exists an afp sequence $\left(z_{n}\right) \subset K$ such that for $i=1,2$ $\lim \sup _{n}\left\|z_{n}-x_{n}^{i}\right\| \leq \frac{1}{2} \lim \sup _{n}\left\|x_{n}^{1}-x_{n}^{2}\right\|$.

Theorem 6. The space $Z$ enjoys the (FPP).

Proof. We shall argue by contradiction using as our main tool the well known Goebel-Karlovitz lemma $[26,27]$ whose statement is as follows: Let $X$ be a Banach space and $T: K \rightarrow K$ be a nonexpansive mapping, where $K$ is a minimal nonempty $T$-invariant convex subset of $X$. If $K$ is weakly compact and $\left(x_{n}\right)$ is an afps for $T$, then for any $x \in K, \lim _{n}\left\|x_{n}-x\right\|=\operatorname{diam} K$, where $\operatorname{diam} K$ is the diameter of $K$. From this it follows that there exists a subsequence $\left(x_{n_{k}}\right)$ of $\left(x_{n}\right)$ such that $\lim _{k}\left\|x_{n_{k}}-x_{n_{k+1}}\right\|=\operatorname{diamK}$.

So suppose that $Z$ lacks the (FPP). Then there exists a nonexpansive mapping $T: K$ $\rightarrow K$ where $K \subset Z$ is a nonempty weakly compact convex set and $T$ does not have a fixed point. Let us assume that $K$ is minimal for $T$. Let $\left(x_{n}\right) \subset K$ be an afps for $T$. By standard arguments, we may suppose that $0 \in K, x_{n} \underset{w}{\rightarrow} 0, \operatorname{diam} K=1$. By passing to an appropriate subsequence of $\left(x_{n}\right)$, still called $\left(x_{n}\right)$, we may suppose that there is a basic block sequence $\left(u_{n}\right)$ such that $u_{1}(1)=u_{1}(2)=0, \lim _{n}|| x_{n}-u_{n} \|=0$ and, if supp $u_{n}=$ $\left\{i: u_{n}(i) \neq 0\right\}$, then max supp $u_{n}+4<\min \operatorname{supp} u_{n+1}$. By taking another appropriate subsequence we may suppose further

i) $\lim _{n}\left\|P x_{n}\right\|, \lim _{n}\left\|P x_{n}\right\|_{2}, \lim _{n}|| Q x_{n} \|_{2}$ exist.

By passing to another subsequence, we also assume, by the Goebel- Karlovitz lemma, that $\left\|x_{n}-x_{n+1}\right\| \rightarrow 1$.

Let $x_{n}^{1}=x_{2 n-1}, x_{n}^{2}=x_{2 n}$. By Lemma 1 , there exists an afps $\left(z_{n}\right)$ such that $\lim \sup _{n}\left\|z_{n}-x_{n}^{i}\right\| \leq \frac{1}{2} \lim \sup _{n}\left\|x_{n}^{1}-x_{n}^{2}\right\| \leq \frac{1}{2}$.

If we take any subsequence $\left(z_{n_{k}}\right)$, since $x_{n_{k}}^{1}=x_{2 n_{k}-1}$ and $x_{n_{k}}^{2}=x_{2 n_{k}}$, we get that $\lim _{k}\left\|x_{n_{k}}^{1}-x_{n_{k}}^{2}\right\|=1$. Hence for $i=1,2$ we must have that $\lim _{k}\left\|z_{n_{k}}-x_{n_{k}}^{i}\right\|=\frac{1}{2}$. For convenience we call the afps $\left(z_{n_{k}}\right)_{k}$ and $\left(x_{n_{k}}^{i}\right)_{k}$ again $\left(z_{k}\right)$ and $\left(x_{k}^{i}\right)$. By passing to appropriate subsequences of $\left(z_{n}\right)$ we finally get afp sequences $\left(x_{n}^{1}\right),\left(x_{n}^{2}\right)$ and $\left(z_{n}\right)$ such that

(a) $\lim _{n}\left\|P x_{n}^{1}\right\|=\lim _{n}\left\|P x_{n}^{2}\right\|, \lim _{n}\left\|P x_{n}^{1}\right\|_{2}=\lim _{n}\left\|P x_{n}^{2}\right\|_{2}, \lim _{n}\left\|Q x_{n}^{1}\right\|_{2}=\lim _{n}\left\|Q x_{n}^{2}\right\|_{2}$.

(b) $\lim _{n}\left\|x_{n}^{1}-x_{n}^{2}\right\|=1$. 
(c) $\lim _{n}\left\|P z_{n}\right\|, \lim _{n}\left\|P z_{n}\right\|_{2}, \lim _{n}\left\|Q z_{n}\right\|_{2}$ exist.

(d) $\lim _{n}\left\|z_{n}-x_{n}^{i}\right\|=\frac{1}{2}$ for $i=1,2$.

(e) $\lim _{n}\left\|P\left(z_{n}-x_{n}^{i}\right)\right\|, \lim _{n}\left\|P\left(z_{n}-x_{n}^{i}\right)\right\|_{2}, \lim _{n}\left\|Q\left(z_{n}-x_{n}^{i}\right)\right\|_{2}$ exist.

(f) $\quad \lim _{n}\left\|x_{n}^{1}+x_{n}^{2}-z_{n}\right\|, \quad \lim _{n}\left\|P\left(x_{n}^{1}+x_{n}^{2}-z_{n}\right)\right\|, \quad \lim _{n}\left\|P\left(x_{n}^{1}+x_{n}^{2}-z_{n}\right)\right\|_{2} \quad$ and $\lim _{n}\left\|Q\left(x_{n}^{1}+x_{n}^{2}-z_{n}\right)\right\|_{2}$ exist.

Consequently, again appealing to the Goebel- Karlovitz lemma,

$$
1=\lim _{n}\left\|x_{n}^{2}\right\| \leq \lim _{n}\left\|z_{n}-x_{n}^{1}\right\|+\lim _{n}\left\|x_{n}^{1}+x_{n}^{2}-z_{n}\right\|=\frac{1}{2}+\lim _{n}\left\|x_{n}^{1}+x_{n}^{2}-z_{n}\right\| .
$$

Thus $\lim _{n}\left\|x_{n}^{1}+x_{n}^{2}-z_{n}\right\| \geq \frac{1}{2}$. Hence

$$
\lim _{n}\left\|x_{n}^{1}+x_{n}^{2}-z_{n}\right\|_{2}=a>0 \text {. }
$$

Now let $u_{n}^{1}=u_{2 n-1}, u_{n}^{2}=u_{2 n}$.

By the assumption on the supports of $u_{n}^{1}$ and $u_{n}^{2}$, for fixed $n$ and for any $j \in \mathbb{N}$, there exists $r(n, j) \in\{1,2\}$ such that $1,2 j+1,2 j+3$ do not belong to supp $u_{n}^{r(n, j)}$. But then

$$
\begin{aligned}
& \quad \frac{1}{3}\left\|P\left(z_{n}-u_{n}^{r(n, j)}\right)\right\|_{2}+\left|z_{n}(1)+z_{n}(2 j+1)+z_{n}(2 j+3)\right|= \\
& \quad \frac{1}{3}\left\|P\left(z_{n}-u_{n}^{r(n, j)}\right)\right\|_{2}+\mid\left(z_{n}-u_{n}^{r(n, j)}\right)(1)+\left(z_{n}-u_{n}^{r(n, j)}\right)(2 j+1) \\
& \quad+\left(z_{n}-u_{n}^{r(n, j)}\right)(2 j+3) \mid \leq\left\|P\left(z_{n}-u_{n}^{r(n, j)}\right)\right\|+\left\|Q\left(z_{n}-u_{n}^{r(n, j)}\right)\right\| \\
& =\left\|z_{n}-u_{n}^{r(n, j)}\right\| .
\end{aligned}
$$

Thus,

$$
\begin{array}{r}
\left|z_{n}(1)+z_{n}(2 j+1)+z_{n}(2 j+3)\right| \leq \\
\left\|z_{n}-u_{n}^{r(n, j)}\right\|-\frac{1}{3}\left\|P\left(z_{n}-u_{n}^{r(n, j)}\right)\right\|_{2}
\end{array}
$$

and similarly for any $k \in \mathbb{N}$, there exists $s(n, k) \in\{1,2\}$ such that $1,2 k, 2 k+2$ do not belong to supp $u_{n}^{s(n, k)}$ and

$$
\begin{array}{r}
\left|z_{n}(2)+z_{n}(2 k)+z_{n}(2 k+2)\right| \leq \\
\left\|z_{n}-u_{n}^{s(n, k)}\right\|-\frac{1}{3}\left\|Q\left(z_{n}-u_{n}^{s(n, k)}\right)\right\|_{2} .
\end{array}
$$

Therefore,

$$
\begin{aligned}
& \quad \lim \sup _{n}\left|z_{n}(1)+z_{n}(2 j+1)+z_{n}(2 j+3)\right| \leq \frac{1}{2} \\
& \quad \text { and } \limsup _{n}\left|z_{n}(2)+z_{n}(2 k)+z_{n}(2 k+2)\right| \leq \frac{1}{2} .
\end{aligned}
$$


Observe that, since the $u_{n}^{i}$ have disjoint supports, the inner product $\left\langle u_{n}^{1}, u_{n}^{2}\right\rangle=0$, and

$$
\begin{gathered}
\left\|z_{n}\right\|_{2}^{2}=\left\|z_{n}-u_{n}^{1}\right\|_{2}^{2}+\left\|z_{n}-u_{n}^{2}\right\|_{2}^{2} \\
-\left\|u_{n}^{1}+u_{n}^{2}-z_{n}\right\|_{2}^{2}+2\left\langle u_{n}^{1}, u_{n}^{2}\right\rangle= \\
\left\|z_{n}-u_{n}^{1}\right\|_{2}^{2}+\left\|z_{n}-u_{n}^{2}\right\|_{2}^{2}-\left\|u_{n}^{1}+u_{n}^{2}-z_{n}\right\|_{2}^{2} .
\end{gathered}
$$

The above also implies

$$
\begin{aligned}
& \left\|P z_{n}\right\|_{2}^{2} \\
= & \left\|P\left(z_{n}-u_{n}^{1}\right)\right\|_{2}^{2}+\left\|P\left(z_{n}-u_{n}^{2}\right)\right\|_{2}^{2}-\left\|P\left(u_{n}^{1}+u_{n}^{2}-z_{n}\right)\right\|_{2}^{2} .
\end{aligned}
$$

By (4.2) and by $\mathrm{d}$ ), for $i=1,2$ it holds that $\lim _{n}\left\|z_{n}-u_{n}^{i}\right\|_{2}^{2} \leq \frac{9}{4}$. From this and (4.8), we obtain for $a_{n}=\left\|u_{n}^{1}+u_{n}^{2}-z_{n}\right\|_{2}$, that if $\lim _{n} a_{n}=a$, where by (4.4) $a>0$,

$$
\lim _{n}\left(\left\|P z_{n}\right\|_{2}^{2}+\left\|Q z_{n}\right\|_{2}^{2}\right)^{\frac{1}{2}}=\lim _{n}\left\|z_{n}\right\|_{2} \leq\left(\frac{18}{4}-a^{2}\right)^{\frac{1}{2}} .
$$

There are three possible cases: (I) There exists a subsequence of $\left(z_{n}\right)$ with $\left\|z_{n_{k}}\right\|=\frac{1}{3}\left(\left\|P z_{n_{k}}\right\|_{2}+\left\|Q z_{n_{k}}\right\|_{2}\right)$, (II) there exists a subsequence such that $\left\|z_{n_{k}}\right\|=\frac{1}{3}\left\|P z_{n_{k}}\right\|_{2}+\left|z_{n_{k}}(1)+z_{n_{k}}\left(2 j_{k}+1\right)+z_{n_{k}}\left(2 j_{k}+3\right)\right|$ for some sequence $\left(j_{k}\right)$ in $\mathbb{N}$ (or $\left\|z_{n_{k}}\right\|=\left|z_{n_{k}}(2)+z_{n_{k}}\left(2 j_{k}\right)+z_{n_{k}}\left(2 j_{k}+2\right)\right|+\frac{1}{3}\left\|Q z_{n_{k}}\right\|_{2}$ for some sequence $\left(j_{k}\right)$ in $\left.\mathbb{N}\right)$ or (III) there exist $\left(j_{n}\right)$ and $\left(k_{n}\right)$ so that

$$
\left\|z_{n}\right\|=\left|z_{n}(1)+z_{n}\left(2 j_{n}+1\right)+z_{n}\left(2 j_{n}+3\right)\right|+\left|z_{n}(2)+z_{n}\left(2 k_{n}\right)+z_{n}\left(2 k_{n}+2\right)\right| .
$$

In any of those cases we will call the subsequence $\left(z_{n}\right)$ again. Again, by the GoebelKarlovitz lemma,

$$
\lim _{n}\left\|z_{n}\right\|=1
$$

(I) By (4.10) $\lim _{n} \frac{1}{3}\left(\left\|P z_{n}\right\|_{2}+\left\|Q z_{n}\right\|_{2}\right) \leq \frac{\sqrt{2}}{3}\left(\frac{9}{2}-a^{2}\right)^{\frac{1}{2}}=\left(1-\frac{2 a^{2}}{9}\right)<1$. So $\left(z_{n}\right)$ cannot be an afps.

(II) Now suppose that $\left\|z_{n}\right\|=\frac{1}{3}\left\|P z_{n}\right\|_{2}+\left|z_{n}(1)+z_{n}\left(2 j_{n}+1\right)+z_{n}\left(2 j_{n}+3\right)\right|$ for some sequence $\left(j_{n}\right)$ in $\mathbb{N}$. By passing to a subsequence if necessary we may suppose that $2 j_{n}+1$ and $2 j_{n}+3$ are not in the support of $u_{n}^{1}$ (or of $u_{n}^{2}$ ), Define

$$
\begin{aligned}
& c_{n}=\left\|P u_{n}^{1}+P u_{n}^{2}-P z_{n}\right\|_{2}^{2}, \\
& A_{n}=\left\|P z_{n}-P u_{n}^{1}\right\|_{2}, B_{n}=\left\|P z_{n}-P u_{n}^{2}\right\|_{2}, \\
& D_{n}=\left|z_{n}(1)+z_{n}\left(2 j_{n}+1\right)+z_{n}\left(2 j_{n}+3\right)\right|=\left|\left(z_{n}-u_{n}^{1}\right)(1)+\left(z_{n}-u_{n}^{1}\right)\left(2 j_{n}+1\right)+\left(z_{n}-u_{n}^{1}\right)\left(2 j_{n}+3\right)\right| .
\end{aligned}
$$

If $A_{n}+B_{n}=0$; we have $P z_{n}=P u_{n}^{1}=P u_{n}^{2}$ and this is only possible if $P z_{n}=0$. Hence, if we had $A_{n_{k}}+B_{n_{k}}=0, k=1,2, \ldots$, by (4.7) 


$$
\begin{aligned}
\lim _{n}\left\|z_{n_{k}}\right\| & =\lim _{k}\left(\frac{1}{3}\left\|P z_{n_{k}}\right\|_{2}+\left|z_{n_{k}}(1)+z_{n_{k}}\left(2 j_{n_{k}}+1\right)+z_{n_{k}}\left(2 j_{n_{k}}+3\right)\right|\right) \\
& =\lim _{k}\left|z_{n_{k}}(1)+z_{n_{k}}\left(2 j_{n_{k}}+1\right)+z_{n_{k}}\left(2 j_{n_{k}}+3\right)\right| \leq \frac{1}{2}
\end{aligned}
$$

and again $\left(z_{n_{k}}\right)$ would not be an afps.

Thus we assume that there exists $N_{0}$ so that for $n>N_{0}, A_{n}+B_{n} \neq 0$, then, since $\frac{1}{3} A_{n}+D_{n} \leq\left\|z_{n}-u_{n}^{1}\right\|$, by (4.9),

$$
\begin{aligned}
\lim _{n}\left\|z_{n}\right\|= & \lim _{n}\left(\frac{1}{3} \sqrt{\left\|P z_{n}-P u_{n}^{1}\right\|_{2}^{2}+\left\|P z_{n}-P u_{n}^{2}\right\|_{2}^{2}-c_{n}+}\right. \\
& \left.\left|z_{n}(1)+z_{n}\left(2 j_{n}+1\right)+z_{n}\left(2 j_{n}+3\right)\right|\right) \\
= & \lim _{n}\left(\frac{1}{3} \sqrt{A_{n}^{2}+B_{n}^{2}-c_{n}}+D_{n}\right)= \\
= & \frac{1}{3} \lim _{n}\left(A_{n}+B_{n}-\frac{2 A_{n} B_{n}+c_{n}}{A_{n}+B_{n}+\sqrt{A_{n}^{2}+B_{n}^{2}-c_{n}}}\right)+\lim _{n} D_{n} \\
\leq & \lim _{n}\left(\left\|z_{n}-u_{n}^{1}\right\|+\frac{1}{3} B_{n}-\frac{1}{3} \frac{2 A_{n} B_{n}+c_{n}}{A_{n}+B_{n}+\sqrt{A_{n}^{2}+B_{n}^{2}-c_{n}}}\right) \\
\leq & \lim _{n}\left(\frac{1}{2}+\frac{1}{2}-\frac{1}{3} \frac{2 A_{n} B_{n}+c_{n}}{A_{n}+B_{n}+\sqrt{A_{n}^{2}+B_{n}^{2}-c_{n}}}\right) \leq 1 .
\end{aligned}
$$

We obtain $\lim _{n} \quad\left\|z_{n}\right\|=1$ if and only if a) $\frac{1}{3} \lim _{n} B_{n}=\lim _{n} \frac{1}{3}\left\|P z_{n}-P u_{n}^{2}\right\|_{2}=\frac{1}{2}=\lim _{n}\left\|z_{n}-u_{n}^{2}\right\|$ and b) $\lim _{n} 2 A_{n} B_{n}+c_{n}=0$. Thus from a) $\lim _{n}\left\|Q\left(z_{n}-u_{n}^{2}\right)\right\|=0$. This means that $z_{n}=P z_{n}+Q u_{n}^{2}+v_{n}$ where $v_{n} \rightarrow 0$. From b) $\lim _{n} 2 A_{n} B_{n}=-\lim _{n} c_{n}$ and since all the quantities involved are nonnegative and $\lim _{n} B_{n} \neq 0$, then $\lim _{n} A_{n}=\lim _{n} c_{n}=0$. This implies on one hand that $z_{n}=P u_{n}^{1}+Q z_{n}+w_{n}$ where $w_{n} \rightarrow 0$ and on the other $z_{n}=P u_{n}^{1}+P u_{n}^{2}+Q z_{n}+\tau_{n}$ where $\tau_{n} \rightarrow 0$. Putting all of this together we obtain: $z_{n}=P u_{n}^{1}+Q u_{n}^{2}+\rho_{n}$ where $\rho_{n} \rightarrow 0$ and $P u_{n}^{2} \rightarrow 0$. But then $\left\|Q u_{n}^{2}\right\| \rightarrow 1$ and therefore $P u_{n}^{1} \rightarrow 0$ and $z_{n}=Q u_{n}^{2}+\sigma_{n}$ where $\sigma_{n}$ $\rightarrow 0$. Hence

$$
1=\lim _{n}\left\|u_{n}^{1}-u_{n}^{2}\right\|=\lim _{n}\left\|u_{n}^{1}-P u_{n}^{2}-z_{n}+\sigma_{n}\right\|=\lim _{n}\left\|z_{n}-u_{n}^{1}\right\|=\frac{1}{2}
$$

and this is a contradiction. The remaining cases are done similarly.

(III) Last suppose that there exist $\left(j_{n}\right)$ and $\left(k_{n}\right)$ so that

$$
\begin{aligned}
\left\|z_{n}\right\| & =\left|z_{n}(2)+z_{n}\left(2 k_{n}\right)+z_{n}\left(2 k_{n}+2\right)\right|+\left|z_{n}(1)+z_{n}\left(2 j_{n}+1\right)+z_{n}\left(2 j_{n}+3\right)\right| \\
& =\left\|P z_{n}\right\|+\left\|Q z_{n}\right\| .
\end{aligned}
$$

Then, by (4.7) $\lim _{n}\left\|P z_{n}\right\| \leq \frac{1}{2}$ and also $\lim _{n}\left\|Q z_{n}\right\| \leq \frac{1}{2}$. So, in order for $\lim _{n}\left\|z_{n}\right\|$ $=1$, it is necessary that

$$
\lim _{n}\left\|P z_{n}\right\|=\frac{1}{2} \text { and } \lim _{n}\left\|Q z_{n}\right\|=\frac{1}{2} \text {. }
$$


Assume that for a subsequence called $\left(z_{n}\right)$ again, $2 j_{n}+1,2 j_{n}+3$ are not in the support of $u_{n}^{1}$ and suppose first that $2 k_{n}$ and $2 k_{n}+2$ do not belong to the support of $u_{n}^{1}$. Then $\left|z_{n}(2)+z_{n}\left(2 k_{n}\right)+z_{n}\left(2 k_{n}+2\right)\right|+\left|z_{n}(1)+z_{n}\left(2 j_{n}+1\right)+z_{n}\left(2 j_{n}+3\right)\right| \leq\left\|z_{n}-u_{n}^{1}\right\|$ and since $\lim _{n}\left\|z_{n}-u_{n}^{1}\right\|=\frac{1}{2}$, in this case $\lim _{n}\left\|z_{n}\right\| \leq \frac{1}{2}$.

Thus suppose that either $2 k_{n}$ or $2 k_{n}+2$ belongs to the support of $u_{n}^{1}$ and hence $\left\{2 k_{n}, 2 k_{n}+2\right\}$ is disjoint with the support of $u_{n}^{2}$. We have by (4.12),

$$
\begin{aligned}
& \frac{1}{2}=\lim _{n}\left|z_{n}(2)+z_{n}\left(2 k_{n}\right)+z_{n}\left(2 k_{n}+2\right)\right| \leq \\
& \lim _{n}\left(\left\|z_{n}-u_{n}^{2}\right\|-\frac{1}{3}\left\|Q\left(z_{n}-u_{n}^{2}\right)\right\|_{2}\right) \leq \frac{1}{2},
\end{aligned}
$$

and

$$
\begin{gathered}
\frac{1}{2}=\lim _{n}\left|z_{n}(1)+z_{n}\left(2 j_{n}+1\right)+z_{n}\left(2 j_{n}+3\right)\right| \leq \\
\lim _{n}\left(\left\|z_{n}-u_{n}^{1}\right\|-\frac{1}{3}\left\|P\left(z_{n}-u_{n}^{1}\right)\right\|_{2}\right) \leq \frac{1}{2} .
\end{gathered}
$$

Thus $\lim _{n}\left\|P\left(z_{n}-u_{n}^{1}\right)\right\|_{2}=0$ and $\lim _{n}\left\|Q\left(z_{n}-u_{n}^{2}\right)\right\|_{2}=0$. Hence, since $\|\cdot\| \cdot$ and $\|\cdot\|_{2}$ are equivalent, we get $\lim _{n}\left\|P\left(z_{n}-u_{n}^{1}\right)\right\|=0, \lim _{n}\left\|Q\left(z_{n}-u_{n}^{2}\right)\right\|=0$ and

$$
\lim _{n}\left\|P z_{n}\right\|=\lim _{n}\left\|P u_{n}^{1}\right\|=\frac{1}{2} \text { and } \lim _{n}\left\|Q z_{n}\right\|=\lim _{n}\left\|Q u_{n}^{2}\right\|=\frac{1}{2} .
$$

By a) $\lim _{n}\left\|Q u_{n}^{1}\right\|=\frac{1}{2}$ and $\lim _{n}\left\|P u_{n}^{2}\right\|=\frac{1}{2}$ also. Now let

$$
y_{n}^{1}=x_{n}^{1}, y_{n}^{2}=z_{n}, v_{n}^{1}=u_{n}^{1}=P u_{n}^{1}+Q u_{n}^{1}, v_{n}^{2}=P u_{n}^{1}+Q u_{n}^{2} .
$$

Then $\left(y_{n}^{1}\right)$ and $\left(y_{n}^{2}\right)$ are two afp sequences in $K$.

By the above, $\quad \lim _{n}\left\|y_{n}^{1}-v_{n}^{1}\right\|=\lim _{n}\left\|y_{n}^{2}-v_{n}^{2}\right\|=0 . \quad$ Also, $\lim _{n}\left\|y_{n}^{1}-y_{n}^{2}\right\|=\lim _{n}\left\|x_{n}^{1}-z_{n}\right\|=\frac{1}{2}$. Applying Lemma 1 there exists an afps $\left(w_{n}\right) \subset K$ such that for $i=1,2$ we have $\lim \sup _{n}\left\|w_{n}-v_{n}^{i}\right\| \leq \frac{1}{4}$. But since $\lim _{n}\left\|v_{n}^{1}-v_{n}^{2}\right\|=\lim _{n}\left\|y_{n}^{1}-y_{n}^{2}\right\|=\frac{1}{2}$, we get that for $i=1,2$

$$
\lim _{n}\left\|w_{n}-v_{n}^{i}\right\|=\frac{1}{4}
$$

We may assume by passing to a subsequence, that $\lim _{n}|| P w_{n}\left\|, \lim _{n}|| P w_{n}\right\|_{2}, \lim \|$ $Q w_{n} \|_{2}$ and for $i=1,2, \lim _{n}\left\|P\left(w_{n}-v_{n}^{i}\right)\right\|, \lim _{n}\left\|P\left(w_{n}-v_{n}^{i}\right)\right\|_{2}, \lim _{n}\left\|Q\left(w_{n}-v_{n}^{i}\right)\right\|_{2}$ all exist. Then

$$
\begin{aligned}
\lim _{n}\left\|P w_{n}\right\| & \leq \lim _{n}\left(\left\|P w_{n}-P v_{n}^{1}\right\|+\left\|P v_{n}^{1}\right\|\right) \\
& \leq \frac{1}{4}+\lim _{n}\left\|P u_{n}^{1}\right\|=\frac{3}{4} .
\end{aligned}
$$

From $\quad \lim _{n}\left\|u_{n}^{1}+u_{n}^{2}\right\|=\lim _{n}\left\|u_{n}^{1}-u_{n}^{2}\right\|=1, \quad \lim _{n}\left\|Q u_{n}^{1}+Q u_{n}^{2}\right\| \geq \lim _{n}\left\|Q u_{n}^{1}\right\|=\frac{1}{2}$, $\lim _{n}\left\|P u_{n}^{1}+P u_{n}^{2}\right\| \geq \lim _{n}\left\|P u_{n}^{1}\right\|=\frac{1}{2}$, we obtain $\lim _{n}\left\|Q u_{n}^{1}+Q u_{n}^{2}\right\|=\lim _{n}\left\|P u_{n}^{1}+P u_{n}^{2}\right\|=\frac{1}{2}$. 
Similarly $\lim _{n}\left\|Q u_{n}^{1}-Q u_{n}^{2}\right\|=\lim _{n}\left\|P u_{n}^{1}-P u_{n}^{2}\right\|=\frac{1}{2}$. Then

$$
\begin{aligned}
& \lim _{n}\left\|Q w_{n}\right\| \leq \lim _{n}\left(\left\|\frac{Q w_{n}-Q v_{n}^{1}}{2}\right\|+\left\|\frac{Q w_{n}-Q v_{n}^{2}}{2}\right\|+\left\|\frac{Q v_{n}^{1}+Q v_{n}^{2}}{2}\right\|\right) \\
& \leq \frac{1}{8}+\frac{1}{8}+\lim _{n}\left\|\frac{Q u_{n}^{1}+Q u_{n}^{2}}{2}\right\| \leq \frac{1}{4}+\frac{1}{2} \frac{1}{2}=\frac{1}{2} \text {. }
\end{aligned}
$$

Also, since $\left\langle\mathrm{Q} v_{n}^{1}, \mathrm{Q} v_{n}^{2}\right\rangle=\left\langle\mathrm{Q} u_{n}^{1}, \mathrm{Q} u_{n}^{2}\right\rangle=0$,

$$
\begin{aligned}
\lim _{n}\left\|Q w_{n}\right\|_{2}^{2}= & \lim _{n}\left(\left\|Q\left(w_{n}-v_{n}^{1}\right)\right\|_{2}^{2}+\left\|Q\left(w_{n}-v_{n}^{2}\right)\right\|_{2}^{2}\right. \\
& \left.-\left\|Q\left(v_{n}^{1}+v_{n}^{2}-w_{n}\right)\right\|_{2}^{2}+2\left\langle Q v_{n}^{1}, Q v_{n}^{2}\right\rangle\right) \\
\leq & 9 \frac{1}{16}+9 \frac{1}{16}=\frac{9}{8} .
\end{aligned}
$$

By a) there exists $c$ such that $\lim _{n}\left\|P u_{n}^{1}\right\|_{2}=\lim _{n}\left\|P u_{n}^{2}\right\|_{2}=c$ and since $\lim _{n}\left\|P u_{n}^{1}\right\|=\frac{1}{2}$, we get that $c>0$. Further,

$$
\begin{aligned}
\frac{1}{2} & \geq \frac{1}{3} \lim _{n}\left\|P\left(u_{n}^{1}-u_{n}^{2}\right)\right\|_{2} \\
& =\frac{1}{3} \lim _{n} \sqrt{\left\|P u_{n}^{1}\right\|_{2}^{2}+\left\|P u_{n}^{2}\right\|_{2}^{2}}=\frac{\sqrt{2}}{3} c .
\end{aligned}
$$

Hence $\lim _{n}\left\|P u_{n}^{i}\right\|_{2} \leq \frac{3}{2 \sqrt{2}}$ and

$$
\begin{aligned}
\lim _{n}\left\|P w_{n}\right\|_{2}^{2}= & \lim _{n}\left(\left\|P\left(w_{n}-v_{n}^{1}\right)\right\|_{2}^{2}+\left\|P\left(w_{n}-v_{n}^{2}\right)\right\|_{2}^{2}\right. \\
& \left.-\left\|P\left(v_{n}^{1}+v_{n}^{2}-w_{n}\right)\right\|_{2}^{2}+2\left\langle P v_{n}^{1}, P v_{n}^{2}\right\rangle\right) \\
\leq & 9 \frac{1}{16}+9 \frac{1}{16}+2 \lim _{n}\left\|P u_{n}^{1}\right\|_{2}^{2}=\frac{9}{8}+\frac{18}{8}=\frac{27}{8}
\end{aligned}
$$

and therefore $\frac{1}{3} \lim _{n}\left\|P w_{n}\right\|_{2} \leq \sqrt{\frac{3}{8}}$. Hence by (4.14)

$$
\lim _{n} \frac{1}{3}\left(\left\|P w_{n}\right\|_{2}+\left\|Q w_{n}\right\|_{2}\right) \leq \frac{1+\sqrt{3}}{\sqrt{8}}<1 \text {. }
$$

As before, since the supports of $\mathrm{Q} v_{n}^{1}$ and $\mathrm{Q} v_{n}^{2}$ are disjoint, for $j$ there exists $i$ such that $2 j+1,2 j+3$ do not belong to the support of $Q v_{n}^{i}$ Suppose that $\left\|w_{n}\right\|=\left\|P w_{n}\right\|$ $+\left|w_{n}(1)+w\left(2 j_{n}+1\right)+w_{n}\left(2 j_{n}+3\right)\right|$ and that for a subsequence of $\left(w_{n}\right)$ we have $2 j_{n}+$ $1,2 j_{n}+3$ do not belong to the support of $Q v_{n}^{1}$ and $\lim _{n} \mid w_{n}(1)+w\left(2 j_{n}+1\right)+w_{n}\left(2 j_{n}+\right.$ 3)| exists. As usual call it $\left(w_{n}\right)$ again. Then

$$
\begin{aligned}
& \frac{1}{3} \lim _{n}\left\|P\left(w_{n}-v_{n}^{1}\right)\right\|_{2}+\lim _{n}\left|w_{n}(1)+w\left(2 j_{n}+1\right)+w_{n}\left(2 j_{n}+3\right)\right| \\
\leq & \lim _{n}\left\|w_{n}-v_{n}^{1}\right\| \leq \frac{1}{4} .
\end{aligned}
$$

Thus, if $\lim _{n} \frac{1}{3}\left\|P\left(w_{n}-v_{n}^{1}\right)\right\|_{2}=a>0$, by (4.13)

$$
\lim _{n}\left\|P w_{n}\right\|+\lim _{n}\left|w_{n}(1)+w\left(2 j_{n}+1\right)+w_{n}\left(2 j_{n}+3\right)\right| \leq \frac{3}{4}+\frac{1}{4}-a<1 .
$$


If $\lim _{n}\left\|P\left(w_{n}-v_{n}^{1}\right)\right\|=0$, then by (4.13), $\lim _{n}\left\|P w_{n}\right\|=\lim _{n}\left\|P v_{n}^{1}\right\|=\frac{1}{2}$ and then by (4.16),

$$
\lim _{n}\left(\left\|P w_{n}\right\|+\left|w_{n}(1)+w\left(2 j_{n}+1\right)+w_{n}\left(2 j_{n}+3\right)\right|\right) \leq \frac{1}{2}+\frac{1}{4}<1 .
$$

By (4.15), (4.17) and (4.18) the only remaining possible case is that

$$
\left\|w_{n}\right\|=\left|w_{n}(2)+w_{n}\left(2 i_{n}\right)+w_{n}\left(2 i_{n}+2\right)\right|+\frac{1}{3}\left\|Q w_{n}\right\|_{2}
$$

for some sequence $\left(i_{n}\right)$ in $\mathbb{N}$.

Let $\lim _{m}\left\|Q u_{m}^{1}\right\|_{2}=d>0$ and $\varepsilon>0$ so that $\frac{d^{2}-\varepsilon}{\left(\frac{9}{8}+d^{2}\right)^{\frac{1}{2}}+\frac{3}{\sqrt{8}}+\varepsilon}-\varepsilon=\gamma>0$.

Let $n$ be fixed and define $w_{n}^{r}=\sum_{j=1}^{r} w_{n}(j) e_{j}$. Suppose that $r(n)$ is such that

$$
\max \left\{\left(\left\|Q w_{n}\right\|_{2}^{2}-\left\|Q w_{n}^{r(n)}\right\|_{2}^{2}\right)^{\frac{1}{2}},\left\|Q\left(w_{n}-w_{n}^{r(n)}\right)\right\|_{2}\right\}<\varepsilon .
$$

There is $M(r(n))$ so that for $m>M(r(n)), \min \operatorname{supp}\left(u_{m}^{1}\right)>\max \left(r(n), 2 i_{n}+2\right)$ and

$$
\frac{\left\|Q u_{m}^{1}\right\|_{2}^{2}-\varepsilon}{\left(\frac{9}{8}+\left\|Q u_{m}^{1}\right\|_{2}^{2}\right)^{\frac{1}{2}}+\frac{3}{\sqrt{8}}+\varepsilon}-\varepsilon>\frac{1}{2} \gamma
$$

Then

$$
\left\|P\left(w_{n}-u_{m}^{1}\right)\right\| \geq \max _{i}\left|w_{n}(2)+w_{n}\left(2 i_{n}\right)+w_{n}\left(2 i_{n}+2\right)\right|=\left\|P w_{n}\right\| .
$$

By (4.14) $\left\|Q w_{n}\right\|_{2} \leq \sqrt{\frac{9}{8}+\varepsilon}<\frac{3}{\sqrt{8}}+\varepsilon$ for sufficiently large $n$. Therefore, for such $n$ and $m>M(r(n))$ we obtain

$$
\begin{gathered}
\left\|Q\left(w_{n}-u_{m}^{1}\right)\right\|_{2} \geq\left\|Q\left(w_{n}^{r(n)}-u_{m}^{1}\right)\right\|_{2}-\varepsilon=\left(\left\|Q w_{n}^{r(n)}\right\|_{2}^{2}+\left\|Q u_{m}^{1}\right\|_{2}^{2}\right)^{\frac{1}{2}}-\varepsilon \geq \\
\left\|Q w_{n}\right\|_{2}+\frac{\left(\left\|Q w_{n}\right\|_{2}^{2}-\varepsilon+\left\|Q u_{m}^{1}\right\|_{2}^{2}\right)^{\frac{1}{2}}-\varepsilon \geq}{\left(\left\|Q w_{n}\right\|_{2}^{2}-\varepsilon+\left\|Q u_{m}^{1}\right\|_{2}^{2}\right)^{\frac{1}{2}}+\left\|Q w_{n}\right\|_{2}}-\varepsilon \geq \\
\left\|Q w_{n}\right\|_{2}+\frac{\left\|Q u_{m}^{1}\right\|_{2}^{2}-\varepsilon}{\left(\frac{9}{8}+\left\|Q u_{m}^{1}\right\|_{2}^{2}\right)^{\frac{1}{2}}+\frac{3}{\sqrt{8}}+\varepsilon}-\varepsilon \geq\left\|Q w_{n}\right\|_{2}+\frac{\gamma}{2} .
\end{gathered}
$$


Using (4.20), (4.21) and (4.19),

$$
\begin{aligned}
\left\|w_{n}-u_{m}^{1}\right\| & \geq\left\|P\left(w_{n}-u_{m}^{1}\right)\right\|+\frac{1}{3}\left\|Q\left(w_{n}-u_{m}^{1}\right)\right\|_{2} \\
& \geq\left\|P w_{n}\right\|+\frac{1}{3}\left\|Q w_{n}\right\|_{2}+\frac{\gamma}{6}= \\
& =\left\|w_{n}\right\|+\frac{\gamma}{6} .
\end{aligned}
$$

Hence, for every $n$, since $\left(x_{n}^{1}\right)$ is an afps, $1=\lim _{m}\left\|w_{n}-x_{m}^{1}\right\|=\lim _{m}\left\|w_{n}-u_{m}^{1}\right\| \geq\left\|w_{n}\right\|+\frac{\gamma}{6}$. Thus $\lim \sup _{n}\left\|w_{n}\right\|<1$.

Therefore, $\left(w_{n}\right)$ cannot be an afps. The other case is done similarly and this finishes the proof.

\section{Acknowledgements}

We thank the referees for their detailed reading of this paper and their helpful suggestions. The first author was partially funded by SEP CONACYT grant 102380 and by a sabbatical grant of CONACYT. She also wants to thank the Department of Mathematical Analysis of the University of Valencia for its warm hospitality.

\section{Author details}

'Centro de Investigación en Matemáticas (CIMAT), Jalisco s/n, 36240 Guanajuato (Gto.), México ²Dept. Análisis Matemático, Facultad de Matemáticas, 46100 Burjassot, Valencia, Spain

\section{Authors' contributions}

All authors read and approved the final manuscript.

\section{Competing interests}

The authors declare that they have no competing interests.

Received: 2 November 2011 Accepted: 31 May 2012 Published: 31 May 2012

\section{References}

1. Kirk, WA: A fixed point theorem for mappings which do not increase distances. Am Math Mon. 72, $1004-1006$ (1965). doi:10.2307/2313345

2. Kirk, WA: Some questions in metric fixed point theory. Recent Advances on Metric Fixed Point Theory. University of Sevilla, Seville (1996). Ciencias 48

3. Lin, PK: Unconditional bases and fixed points of nonexpansive mappings. Pacific J Math. 116, 69-76 (1985)

4. Baillon, JB, Schöneberg, R: Asymptotic normal structure and fixed points of nonexpansive mappings. Proc Am Math Soc. 81(2), 257-264 (1981). doi:10.1090/S0002-9939-1981-0593469-1

5. Jiménez-Melado, A: Una propiedad geométrica de los espacios de Banach relacionada con la Teoría del Punto Fijo. Doctoral dissertation. Univ. de Málaga (Spain) (1988)

6. Jiménez-Melado, A, Llorens-Fuster, E: A sufficient condition for the fixed point property. Nonlinear Anal Theory Methods Appl. 20, 849-853 (1993). doi:10.1016/0362-546X(93)90073-2

7. Prus, S, Szczepanik, M: Nearly noncreasy Banach spaces. J Math Anal Appl. 307, 255-273 (2005). doi:10.1016/j. jmaa.2004.10.049

8. Prus, S: Banach spaces which are uniformly noncreasy. Proceedings of the Second World Congress of Nonlinear Analysts (Athens). Nonlinear Anal Theory Methods Appl. 30, 2317-2324 (1997)

9. García Falset, J, Llorens Fuster, E, Mazcuñán Navarro, E: Banach spaces which are r-uniformly noncreasy. Nonlinear Anal. 53, 957-975 (2003). doi:10.1016/50362-546X(03)00030-0

10. Fetter, H, Gamboa de Buen, B, García Falset, J: Banach spaces which are somewhat uniformly noncreasy. J Math Anal Appl. 285, 444-455 (2003). doi:10.1016/S0022-247X(03)00410-4

11. Fetter, H, Gamboa de Buen, B: (r, k, l)-somewhat uniformly noncreasy Banach spaces. International Conference on Fixed Point Theory and Applications. pp. 71-80.Yokohama Publication, Yokohama (2004)

12. Domínguez Benavides, T: A geometrical coefficient implying the fixed point property and stability results. Houst J Math. 22(4), 835-849 (1996)

13. Mazcuñán Navarro, E: Geometría de los Espacios de Banach en Teoría Métrica del Punto Fijo. Doctoral dissertation. University of Valencia (2003)

14. García Falset, J, Llorens Fuster, E, Mazcuñán Navarro, E: Uniformly nonsquare Banach spaces have the fixed point property for nonexpansive mappings. J Funct Anal. 233, 494-514 (2006). doi:10.1016/j.jfa.2005.09.002

15. García Falset, J: The fixed point property in spaces with NUS property. J Math Anal Appl. 215, 532-542 (1997). doi:10.1006/jmaa.1997.5657

16. Fetter, $\mathrm{H}$, Llorens Fuster, E: Comparison of P-convexity, O-convexity and other geometrical properties. Preprint

17. Dowling, PN, Randrianantoanina, B, Turett, B: The fixed point property via dual space properties. J Funct Anal. 255, 768-775 (2008). doi:10.1016/j.jfa.2008.04.021

18. Naidu, SVR, Sastry, KPR: Convexity condition in normed spaces. J Reine Angew Math. 297, 35-53 (1978) 
19. Jiménez-Melado, A, Llorens-Fuster, E: The fixed point property in uniformly nonsquare Banach spaces. Boll Un Mat Ital. 10(A), 587-595 (1996)

20. Fetter, H, Gamboa de Buen, B: Properties WORTH and WORTH*, $(1+\delta)$-embeddings in Banach spaces with 1unconditional basis and wFPP. Fixed Point Theory Appl 7 (2010). Article ID 342691

21. van Dulst, D: Some geometric properties related to normal structure. In Nonlinear Analysis and Applications. Lecture Notes in Pure and Applied Mathematics, vol. 80, pp. 155-161.Dekker, New York (1982)

22. Kuczumow, T, Reich, S, Schmidt, M: A fixed point property of $I_{1}$ - product spaces. Proc Am Math Soc. 119, 457-463 (1993)

23. Prus, S, Wiśnicki, A: On the fixed point property in direct sums of Banach spaces with strictly monotone norms. Stud Math. 186, 87-99 (2008). doi:10.4064/sm186-1-8

24. Wiśnicki, A: On the fixed points of nonexpansive mappings in direct sums of Banach spaces. Stud Math. 207, 75-84 (2011). doi:10.4064/sm207-1-5

25. Maurey, B: Points fixes des certain faiblement compacts de L'? Seminaire d Analyse Fonctionelle 1980-1981. Ecole Polytechnique, Palaiseau

26. Goebel, K: On the structure of the minimal invariant sets for nonexpansive mappings. Ann Univ Marie CurieSklodowska. 29, 73-77 (1975)

27. Karlovitz, L: Existence of fixed points for nonexpansive mappings in spaces without normal structure. Pacific J Math. 66 , 153-156 (1976)

doi:10.1186/1687-1812-2012-91

Cite this article as: Fetter Nathansky and Llorens-Fuster: A product space with the fixed point property. Fixed Point Theory and Applications 2012 2012:91.

\section{Submit your manuscript to a SpringerOpen ${ }^{\odot}$} journal and benefit from:

- Convenient online submission

- Rigorous peer review

- Immediate publication on acceptance

- Open access: articles freely available online

- High visibility within the field

- Retaining the copyright to your article

Submit your next manuscript at $\gg$ springeropen.com 